\title{
LIS1 and dynein motor function in neuronal migration and development
}

\author{
Anthony Wynshaw-Boris ${ }^{1}$ and Michael J. Gambello \\ Departments of Pediatrics and Medicine, University of California, San Diego, School of Medicine, \\ La Jolla, California 92093-0627, USA
}

Neuronal migration has been studied extensively for over 30 years in diverse mammalian species from the mouse to human. The sequence of events that occurs during cortical development is shared by all of these species (for reviews, see Gleeson and Walsh 2000; Walsh and Goffinet 2000). At the time of neurogenesis, neural precursors proliferate and differentiate into young postmitotic neurons. These postmitotic immature neurons migrate from the ventricular zone (VZ) to a layer called the preplate at the surface of the developing cerebral cortex. The first migrating neurons split the preplate and form the cortical plate, which develops into the cortex. As migration from the VZ continues, cortical lamination is established in an inside-out fashion. The earliest-born neurons end up deep in the cortex, as later-born neurons migrate past them toward the pial surface to establish more superficial layers of the cortex. The latest-born neurons reside near the pial surface. In the final stages of cortical development, synaptogenesis and apoptotic elimination of populations of neurons occur. Physically, the migration of neurons require the same three steps necessary for migration of any cell: The extension of the leading edge that explores its environment for attractive and repulsive signals; the movement of the nucleus into the leading process, called nucleokinesis; and the retraction of the trailing process.

The understanding of the process of mammalian neuronal migration resulted primarily from neurobiological studies of brain development in normal mammals and mutant mice such as reeler. These studies provided important insight into the laminar development of the CNS. However, they did not identify the genetic and developmental pathways that regulate neuronal migration. Cloning of disease genes for human neuronal migration defects and mouse mutants have provided critical entry points into these pathways, whereas genetic and cell biological studies have guided our understanding of the function of these gene products in neuronal migration. For example, cloning of the gene mutated in reeler and the identification of its protein product RELN

${ }^{1}$ Corresponding author.

E-MAIL awynshawboris@ucsd.edu; FAX (858) 822-3409.

Article and publication are at www.genesdev.org/cgi/doi/10.1101/ gad.886801. led to the elucidation of a RELN-dependent signal transduction pathway (for recent review, see Rice and Curran 1999|. Other genes have been identified that are responsible for neuronal migration defects in the human (LIS1 and DCX) and mouse (Cdk5 and its required activators p35 and p39), but until recently, the relationship between these genes involved in migration were unknown.

Several recent studies have demonstrated that LIS1, the protein product of a gene mutated in the human neuronal migration defect lissencephaly, binds to and regulates dynein motor function in the cell. These studies place LIS1 in the midst of a well-studied motor complex important for several critical cell functions and perhaps provide a context for integrating several neuronal migration pathways. In this review, we will provide some background on the human and mouse studies that provided the entry points to neuronal migration pathways, the investigation of gene function in model systems, and what these studies tell us about the mechanisms regulating neuronal migration. We will focus on the recent studies of LIS1 function and how they can potentially integrate other known pathways of neuronal migration.

\section{Human lissencephaly and LIS1}

Lissencephaly (smooth brain) is a term used to describe the smooth surface of the cortex seen in human brain malformations caused by certain types of neuronal migration defects. Classical or type I lissencephaly defines a subgroup of human neuronal migration disorders characterized by generalized agyria (absence of gyri) and pachygyria (reduced numbers of broadened gyri), four abnormal cortical layers, enlarged ventricles, and generalized neuronal heterotopias (Barkovich et al. 1991; Dobyns and Truwit 1995; Dobyns et al. 1996). Isolated lissencephaly sequence (ILS) is a heterogeneous disorder consisting of variably severe lissencephaly with no other major malformations, whereas Miller-Dieker syndrome (MDS) consists of a generally more severe classical lissencephaly than ILS, characteristic craniofacial anomalies (microcephaly with bitemporal narrowing, a high forehead, a small nose with anteverted nares, thin vermilion border, and micrognathia), and occasionally other malformations (Dobyns et al. 1984). Children with ILS and MDS are severely retarded, have epilepsy, and usu- 
ally die early in childhood (Dobyns et al. 1992). MDS and some cases of ILS are the result of haploinsufficiency at human chromosome $17 \mathrm{p} 13.3$. Virtually $100 \%$ of the patients with MDS and $40 \%$ of patients with ILS have visible or submicroscopic deletions detectable by FISH (Dobyns et al. 1994; Chong et al. 1997).

\section{Human LIS1 mutations are responsible for neuronal migration defects}

The LIS1 gene was cloned from 17p13.3 and was the first gene identified that was associated with neuronal migration defects (Reiner et al. 1993). LIS1 was disrupted in an ILS patient with a translocation and in several other key MDS patients (Chong et al. 1997). Point mutations and an intragenic deletion in LIS1 were identified in ILS patients who showed no gross structural chromosomal rearrangements (Lo Nigro et al. 1997; Pilz et al. 1998). These studies demonstrate that LIS1 is responsible for classical lissencephaly in patients with ILS and contributes to the migration defects seen in patients with MDS. The more severe neuronal migration phenotype and facial dysmorphisms displayed by MDS patients suggest that genes other than LIS1 are responsible for these phenotypes.

LIS1 consists of seven spaced WD-40 repeats and probably assumes the structure of a "propeller wheel," similar to other WD-40 repeat proteins (Neer et al. 1994; Garcia-Higuera et al. 1996). Bovine and murine LIS1 are almost identical in amino acid sequence. In mammals,
LIS1 has at least two identified functions (Fig. 1). LIS1 is a noncatalytic subunit of platelet-activating factor acethylhydrolase (PAFAH) isoform $\mathrm{Ib}$, an inactivating enzyme for platelet-activating factor or PAF (Hattori et al. 1994), so the formal gene name for LIS1 is PAFAH1B1. Only one nonmammalian homolog of the catalytic subunits of PAFAH1B has so far been identified in Drosophila, but it does not appear to possess PAFAH catalytic activity (Sheffield et al. 2000), suggesting that the PAFAH1B function of LIS1 is a mammalian evolutionary adaptation. In addition, LIS1 has a nonenzymatic function (Fig. 1). It is part of a highly conserved pathway that regulates nuclear migration in fungi (Morris 2000). The LIS1 homolog in Aspergillus, NudF, is part of a signaling pathway that regulates nuclear migration along microtubules via the regulation of dynein motor function. As will be discussed below, this function is conserved in all eukaryotes from fungi to mammals. It is unclear how this function is related to PAFAH1B activity. One hypothesis that could link the enzymatic and nonenzymatic functions of LIS1 is that the hydrolysis of PAF facilitates the release of LIS1 from PAFAH1B so it can interact with other molecules involved in migration, such as dynein motors (Fig. 1).

Dosage-dependent neuronal migration defects in Lis1 mutant mice

Although human studies demonstrated that LIS1 mutations are responsible for lissencephaly, it is not possible

\section{Aspergillus}
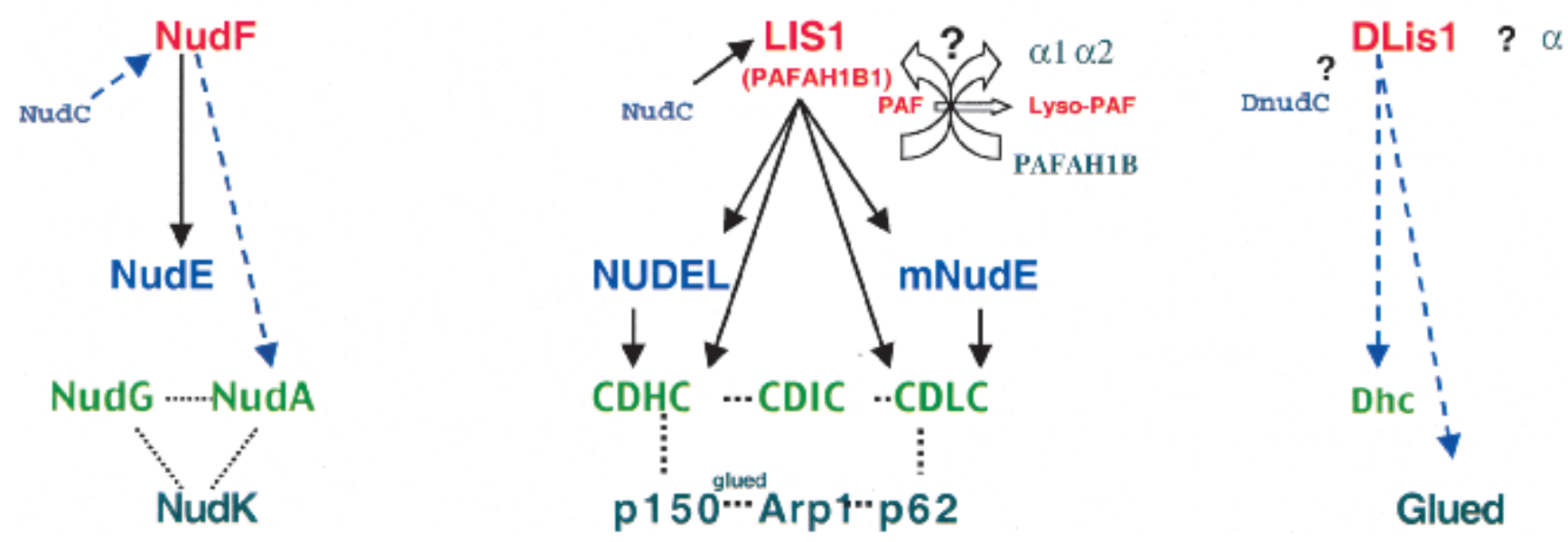

Figure 1. The nuclear distribution pathway first described in Aspergillus nidulans (left) is conserved in mammals (center) and Drosophila (right). Direct interactions of NudF, LIS1, or DLis1 (red) with other proteins are indicated by a black solid arrow. Genetic interactions are represented by a dashed blue arrow. The directions of arrows represent the presumed direction of information transfer. Dotted lines connect proteins in a complex with no attempt to represent the molecular interactions. Genes discovered in an organism with no function assigned are indicated with a question mark. Mammalian LIS1 is part of a complex for an enzyme that inactivates PAF, PAFAH1B. This function appears to be unique to mammalian LIS1. The clear arrows to the right of LIS1 are meant to raise the possibility that PAF binding and inactivation to lyso-PAF by PAFAH1B may release LIS1 from the complex and may provide an added level of regulation of intracellular LIS1 levels. Homologs are in similar colors and assume similar levels in each organism. See text for details. 
to study the neuronal migration defects that occur embryologically during human development. Besides obvious ethical constraints, LIS1 mutations occur de novo in affected children or are the consequence of mild parental mosaicism. To examine the consequences of dosage reduction and complete deficiency of Lis1 in vivo, we produced two knock-out and one conditional knock-out Lis1 mutant alleles by gene targeting in the mouse (Hirotsune et al. 1998). The two knock-out alleles are nulls, while the conditional allele is hypomorphic because of the disruption of transcription or splicing by the insertion of PGKneo in intron 3. By mating mice with various Lis1 alleles, mice were produced with graded reduction in LIS1 dosage. Mice with decreased levels of Lis1 exhibited dose-dependent disorganized cortical layers, hippocampus, and olfactory bulb because of cell autonomous neuronal migration defects (Hirotsune et al. 1998) and are a good model for the human disorder. Complete loss of LIS1 results in peri-implantation lethality, demonstrating that Lis1 is an essential gene. Several lines of evidence support the conclusion that there are in vivo migrational defects in mice with reduction of LIS1 dosage (Hirotsune et al. 1998). These include histological analysis during development, BrdU birthdating experiments, and in vitro migration of granule cells from cerebellar cell reaggregates.

Further studies demonstrated impairments of motor function and cognition in Lis1-null heterozygotes (Paylor et al. 1999), the types of deficits expected based on the human phenotype. The disruption of hippocampal cellular and synaptic physiology was examined in detail in Lis1-deficient mice (Fleck et al. 2000). The hippocampal defects seen in the Lis1 mutant mice were the result of neuronal migration defects, determined by BrdU birthdating studies. Golgi impregnation studies demonstrated that the dendritic arbor of neurons from both wild-type and Lis1+/KO cells located within stratum pyramidale were not significantly different but that the dendritic arbor of heterotopic pyramidal cells was significantly smaller compared to both cell types, with stunted dendrites that make fewer dendritic branches. Electrophysiological studies demonstrated an enhanced excitability in the Lis1+/KO CA1 pyramidal cell layer, disorganized field potentials in the Lis1 mutant hippocampus, and hyperexcitability to electric stimulation. Finally, exposing Lis1 mutant hippocampal slices to conditions of elevated $\left[\mathrm{K}^{+}\right]_{\mathrm{o}}$, the so-called "High- $\mathrm{K}^{+\prime}$ " model of epilepsy, a model for status epilepticus, resulted in increased interictal bursts. These data are consistent with the enhanced excitability and provide a potential explanation for the intractable seizures seen in ILS patients.

\section{Other genes required for neuronal migration}

Several other human and mouse proteins that participate in neuronal migration have been identified either by cloning the human and murine genes responsible for migration defects or on the basis of phenotypes displayed in gene-targeted mice. Some of these genes and pathways with particular relevance to LIS1 will be briefly de- scribed before returning to the functions of LIS1 and what is know about LIS1-dependent pathways.

\section{RELN pathway}

The cloning of RELN, the product of the gene mutated in the most well-known and studied spontaneous recessive mouse mutant reeler (for reviews, see Caviness and Rakic 1978; Goffinet 1984; D'Arcangelo and Curran 1998; Rice and Curran 1999), subsequently led to the delineation of several members of the RELN signaling pathway. RELN is a large extracellular matrix (ECM)-like secreted protein (D'Arcangelo et al. 1995; Hirotsune et al. 1995) produced by the Cajal-Retzius cells lining the outer layer of the preplate (Ogawa et al. 1995). RELN may provide extracellular cues to guide neurons along a RELN-gradient toward the pial surface (Ogawa et al. 1995; D'Arcangelo et al. 1997). RELN binds at least three types of cell surface receptors: the lipoprotein receptors such as VLDLR and ApoER2 (D'Arcangelo et al. 1999; Hiesberger et al. 1999; Trommsdorff et al. 1999), the cadherin-related neuronal receptors (CNRs; Senzaki et al. 1999), and $\alpha 3 \beta 1$-integrin (Dulabon et al. 2000). The relative importance and interactions of these RELN receptors are currently unknown, although RELN binding to $\alpha 3 \beta 1$-integrin is an apparent stop signal for migration (Dulabon et al. 2000). mDab1 (mouse disabled-1), the vertebrate homolog of the Drosophila disabled gene $(d a b)$, transduces the downstream signal of at least some of these RELN receptors. mDab1-deficient mice display a reeler phenotype (Howell et al. 1997a; Sheldon et al. 1997; Ware et al. 1997). mDab1 is a phosphoprotein that binds to a number of tyrosine kinases, including the SH2 domain of c-abl (Howell et al. 1997b), as well as the transmembrane receptor Notch (Giniger 1998). It appears that lipoprotein receptor activation may directly activate mDabl as part of a RELN dependent pathway (D'Arcangelo et al. 1999; Hiesberger et al. 1999), whereas CNR binding to other tyrosine kinases such as fyn may also activate mDab1 (Senzaki et al. 1999). Components downstream of mDabl in the RELN pathway are not known at this time, but this is an area of active investigation.

\section{Cdk5 and its activators}

Homozygous mice with targeted disruption of the serine-threonine kinase Cdk5 (Oshima et al. 1996) or its regulatory subunit p35 (Chae et al. 1997) also demonstrate severe defects in cortical lamination. Though these mice display a reeler-like phenotype, further analysis of the $C d \mathrm{k}^{-/-}$mutants has demonstrated a distinct difference from reeler (Gilmore et al. 1998). In reeler, the preplate is never split by migrating neurons and the cortical neurons remain below it in a disorganized manner. In $C d k 5^{-/-}$mice, the preplate is split by a population of early cortical neurons, but later-born neurons accumulate below this layer. These data suggest that Cdk5 kinase activity is not required for early cortical develop- 
ment but is crucial for migration of later-born neurons. The similar phenotype of Cdk 5 and RELN pathway mutants suggests that many of these proteins participate in a common signal transduction pathway that is crucial for cortical lamination. A recent study identified Cables, a protein that may link the RELN pathway to Cdk5. Cables is an activator of Cdk5 that binds to Cdk5 in the brain along with the c-abl tyrosine kinase (Zukerberg et al. 2000). Active c-abl phosphorylates and activates Cdk5, and Cdk 5 phosphorylation is enhanced by Cables. mDab1 also binds to c-abl and is activated by RELN pathway signals.

\section{Doublecortin}

Although LIS1 was the first identified human gene involved in neuronal migration, others have since been isolated. One of these, doublecortin $(D C X)$, has particular relevance to the pathways discussed in this review. This gene is responsible for $\mathrm{X}$-linked subcortical band heterotopia $\left(\mathrm{SBH}_{\text {; }}\right.$ des Portes et al. 1998; Gleeson et al. 1998). DCX has a predicted kinase domain and may be part of an Abl-dependent transduction pathway involved in neuronal migration (Gleeson et al. 1998). Only males harboring DCX mutations have ILS, as females are mosaics because of X-inactivation and manifest SBH. Recently, it was reported that mutations and deletions of LIS1 or DCX (also known as XLIS) account for $76 \%$ of cases of ILS (des Portes et al. 1998; Pilz et al. 1998). Recent studies have shown that DCX binds to microtubules (Francis et al. 1999; Gleeson et al. 1999; Horesh et al. 1999). In addition, an interaction between DCX and LIS1 was reported (Caspi et al. 2000).

\section{The nuclear distribution (nud) pathway of Aspergillus nidulans}

The relationship among any of the proteins or pathways that participate in mammalian neuronal migration was unclear or unknown until recently. Surprisingly, studies of nuclear distribution mutants in the slime mold Aspergillus nidulans and other related fungi have helped to clarify LIS1-dependent pathways and their function, which in turn led to a more integrated view of neuronal migration pathways.

The biology of nuclear movement has been studied in several fungi including A. nidulans, Saccharomyces cerevisiae, Neurospora crassa, and Nectria haematococca. In the filamentous fungus $A$. nidulans, nuclei migrate toward the growing tip of hyphae to establish regular spacing, a process termed nucleokinesis (Oakley and Morris 1980; Xiang and Morris 1999). A. nidulans is amenable to genetic manipulation, and several nuclear distribution mutants (nud mutants) have been generated to study nucleokinesis in this organism (Morris 1975; Xiang et al. 1999). One of these, nudF, is the fungal homolog of LIS1 (Fig. 1). The nudF gene was isolated as a multicopy suppressor of the nudC mutant that also complemented the nudF6 temperature-sensitive mutant
(Xiang et al. 1995). Like the mammalian protein, NudF contains WD-40 repeats and, remarkably, is $42 \%$ identi$\mathrm{cal}$ and $64 \%$ similar to the human LIS1 protein. NudC is an essential gene in A. nidulans (Chiu et al. 1997). NudC appears to regulate the level of NudF posttranscriptionally, but the biochemical function of NudC is unknown (Osmani et al. 1990). Homologs have been isolated in a number of eukaryotic organisms.

It has long been established that nuclear movement was a microtubule-dependent process (Oakley and Morris 1980, 1981). However, the identification of other nud mutants directly implicated cytoplasmic dynein and dynactin in this process. The nudA and nudG genes code for the heavy chain and the 8-kD light chain of cytoplasmic dynein, respectively (Xiang et al. 1994; Beckwith et al. 1998). A mutant allele of nudF, nudF6, can be suppressed by mutations in $n u d A$, the cytoplasmic dynein heavy chain, providing genetic evidence that NUDA and NUDF are in the same genetic pathway (Willins et al. 1997) and suggests that LIS1 might directly interact with cytoplasmic dynein. A large series of nud mutants has been generated, leading to the identification of at least 10 new loci involved in nucleokinesis, nudI through nudR (Xiang et al. 1999). The nudK gene codes for the actinrelated protein Arp1. This $45-\mathrm{kD}$ protein is part of the dynactin complex, an accessory complex of dynein (Schafer et al. 1994; Schroer 1994). Arp1 is concentrated at the centrosome and, hence, was originally named centractin (Clark and Meyer 1992; Lees-Miller et al. 1992). Dynactin may be a receptor required for dynein-mediated vesicle transport (for review, see Holleran et al. 1998), but the precise mechanism by which dynactin participates in movement of nuclei remains to be determined.

A screen for multicopy suppressors of the nudF phenotype identified nudE (Efimov and Morris 2000). NudE is a homolog of the nuclear distribution protein RO11 in Neurospora crassa (Minke et al. 1999), the mitotic protein MP43 in Xenopus laevis (Stukenberg et al. 1997), and two murine homologs of nudE named NUDEL (for NudE-like) and NudE (see below). The double mutants $n u d F ; n u d E$ and nudE;nudA were similar to the nudF and nudA (cytoplasmic dynein heavy chain) single mutants, indicating that NudF, NudE, and NudA are part of the same genetic pathway (Fig. 1). NudE physically interacts with NudF but not with LIS1. However, chimeric NudE proteins containing the conserved coiled-coil amino terminal domains of human or Xenopus MP43 did physically interact with mammalian LIS1.

Thus, several genes responsible for nuclear movement have been isolated from Aspergillus. The nud pathway clearly involves the fungal LIS1 homolog, NudE, cytoplasmic dynein, and dynactin. The nud pathway is remarkably conserved in eukaryotes (Fig. 1). NudF is the LIS1 homolog in A. nidulans. Cytoplasmic dynein and dynactin are conserved throughout eukaryotic evolution. Homologs have been cloned in Drosophila and several mammalian species.

The process of nuclear migration in fungi is strikingly similar to neuronal migration in mammals. Migrating 
neurons initially extend leading process along radial glial fibers. After completion of this process, the nucleus of the migrating neurons must relocate their position from their birthplace to their final destination in the nervous system. LIS1 is required for mammalian neuronal migration in a dosage-sensitive manner (Hirotsune et al. 1998) and for nuclear migration in $A$. nidulans. Nuclear distribution in $A$. nidulans is also similar to nuclear movement and cellular transport in Drosophila. Therefore, it is not surprising that the same molecular mechanism utilizing NUDEL, NudE, LIS1, and cytoplasmic dynein motors may underlie these processes in all eukaryotes.

\section{Drosophila LIS1 (DLis1) mutants and function}

In Drosophila melanogaster, nuclear movement and cellular transport are dependent on microtubules, cytoplasmic dynein, and dynactin. The Drosophila LIS1 homo$\log$, DLis 1 (Fig. 1), encodes a protein that is $70 \%$ identical to LIS1 and $41 \%$ to NudF (Liu et al. 1999). DLis1 is highly expressed in the germarium and the developing oocyte. Homozygous DLis1 mutant embryos hatched normally but soon experienced growth retardation and died, demonstrating a requirement for DLis1 early in development similar to the mouse (Hirotsune et al. 1998). In the normal Drosophila egg chamber, there is a germline stem cell that divides asymmetrically to produce 15 nurse cells and one oocyte. In DLis1 germ-line mutant clones or cysts, an oocyte rarely develops, and there is a reduction in the number of nurse cells. In another study, the DLis1 gene was isolated in an enhancer screen for oocyte determination and nuclear positioning (Swan et al. 1999), as well as for nurse-cell-to-oocyte transport. This is a phenotype similar to that of the Dhc64C cytoplasmic dynein heavy-chain loss-of-function mutant (McGrail and Hays 1997). The pupal lethal DLis1 homozygous flies demonstrate nuclear migration defects in developing third-instar imaginal discs. A similar phenotype was seen in dynactin Glued subunit mutants (Fan and Ready 1997). Furthermore, Drosophila dynein physically interacts with the p150 subunit of dynactin encoded by Glued (McGrail et al. 1995), suggesting that DLis1, cytoplasmic dynein, and dynactin are associated. In support of this hypothesis, the $\mathrm{Dhc}^{6-6}$ gain-of-function allele of cytoplasmic dynein heavy chain dominantly suppressed the DLis1 homozygous phenotype in developing egg chambers, resulting in fertility, proper nuclear positioning, and nearly normal oocyte growth. These results are similar to those in A. nidulans discussed above.

Because of the early embryonic lethality of flies completely deficient for DLis1, it was impossible to address the function of this protein in somatic cells. DLis1 function was further analyzed by completely eliminating DLis1 in somatic clones of the Drosophila nervous system using mosaic analysis with a repressible cell marker (MARCM) technology (Lee and Luo 1999). These studies support a genetic interaction between DLis1 and cytoplasmic dynein heavy chain in neuroblast proliferation, dendritic growth, and axonal transport (Liu et al. 2000). DLis1 is highly expressed in the developing fly brain and ventral nerve cord, so the analysis was focused on the developing mushroom bodies in the Drosophila central nervous system. Mutant DLis1 mushroom bodies exhibited neuroblast proliferation defects and reduced dendritic arborization, similar to the dendritic arborization defects seen in the mouse. Similar phenotypes were observed in Dhc64C (cytoplasmic dynein heavy chain) lossof-function MARCM clones, supporting the genetic interaction between DLis1 and cytoplasmic dynein initially suggested by egg cell chamber phenotypes (see below). DLis1 mutant neurons developed axonal swellings that suggest a defect in axonal transport, and similar swellings were observed in the Dhc64C MARCM clones. These elegant genetic experiments demonstrate a role for DLis1 in neuroblast proliferation, dendritic elaboration, and axonal transport in Drosophila. The importance of the microtubule cytoskeleton in these processes and the similarities between the DLis1 and Dhc64C mutant MARCM clones further supports a mechanistic association among DLis1, microtubules, cytoplasmic dynein, and dynactin.

Other experiments in Drosophila suggest that DLis1 plays a role in regulating dynein localization (Swan et al. 1999). In stage 5 of oogenesis, DLis1 is concentrated along the cortex of wild-type oocytes. This localization pattern is unchanged in dynein mutants. However, later in development, the oocyte-specific localization of dynein is abolished in egg chambers of the DLis1 mutant, demonstrating that DLis1 is important for the proper localization of dynein in the developing egg chamber. LIS1 binds the pleckstrin homology domain of $\beta$-spectrin, a membrane protein (Wang et al. 1995). In DLis1 mutant egg cell chambers, there are aberrantly formed fusomes or cytoskeletal branched structures composed of membrane skeletal proteins $\alpha$ spectrin, $\beta$ spectrin, ankyrin, and Hu-li tai shao (Hts; Lin et al. 1994; de Cuevas et al. 1996). Mutations in Hts or $\alpha$ spectrin eliminate fusomes and cause egg chambers with $<16$ cells, often with a missing oocyte (Yue and Spradling 1992; de Cuevas et al. 1996). The similarity of this and DLis1 phenotypes suggest a possible interaction between DLis1 and membrane cytoskeletal proteins and may explain the defects seen in the egg chambers of DLis1 mutants.

These studies in Drosophila demonstrate several levels of interaction between DLis1, cytoplasmic dynein, and dynactin in various cellular processes, including nuclear movement in photoreceptor cells, oocyte development, neuroblast proliferation, dendritic growth, and axonal transport.

\section{LIS1 regulates dynein motor function in mammals}

The regulation of dynein motor function by LIS1 and microtubule organization is conserved in mammalian cells. It was shown previously that LIS1 associates with the microtubule fraction of cell supernatants (Sapir et al. 1997). Consistent with these findings, Smith et al. (2000) found that LIS1 is present in regions of high microtubule density in fibroblasts, especially at the centrosome and microtubule organizing center (MTOC). LIS1 colocalized 
with the dynein intermediate chain (IC) and the dynactin subunit $\mathrm{p} 150^{\text {glued }}$ in the ventricular zone and the cortical plate during brain development, and these three proteins were present in a complex in the brain. LIS1 was $\sim 15 x$ more abundant in neurons than Cos-7 cells. Overexpression of LIS1 in COS-7 cells resulted in an increase in LIS1 association with p150'slued and DIC and increased the signal intensity of LIS1 at the MTOC as well as the intensity of $150^{\text {glued }}$ and DIC, suggesting that LIS1 may regulate the distribution of these molecules by increasing retrograde dynein transport. Overexpression of LIS1 also resulted in the peripheral redistribution of microtubules and tighter packing of the Golgi complex around the nucleus. In contrast, reduction of LIS1 in Lis1 +/KO cells resulted in a dispersal of $\mathrm{p} 150^{\text {glued }}$ throughout the cell, enrichment of microtubules near the nucleus, and looser packing of the Golgi complex. Each of these processes is dependent on cytoplasmic dynein motor function and provides strong evidence that LIS1 dosage regulates dynein motor function in fibroblasts. It appears that high expression of LIS1 stimulates the plus-end-directed peripheral movement of microtubule segments by stationary cytoplasmic dynein-dynactin complexes (perhaps located at the cell cortex) as well as the retrograde transport toward the MTOC and centrosome of organelles such as the Golgi complex by mobile dynein-dynactin motors. Reduced levels of LIS1 result in the opposite phenotype.

LIS1 interaction with cytoplasmic dynein and dynactin appears to be important for mitotic cell division and cytokinesis (Faulkner et al. 2000). LIS1 was coimmunoprecipitated from bovine brain cytosol with antibodies to cytoplasmic dynein heavy chain and the dynein intermediate chain, as well as the p150 ${ }^{\text {glued }}, \mathrm{p} 62$, and Arp1 subunits of dynactin. LIS1 was localized at mitotic spindle poles, cell cortex, and kinetochores in cells, and it may have a function during mitosis. Perturbation of LIS1 levels in cultured cells disrupted mitotic progression, mitotic-spindle orientation, and chromosome attachment. Overexpression of LIS1 in MDCK cells increased the number of cells in mitosis and prometaphase, similar to overexpression of dynamitin, a subunit of dynactin. These LIS1-overexpressing cells contained multipolar spindles and unaligned chromosomes, which indicates a defect at the kinetochore. $\mathrm{p} 150^{\text {glued }}$ staining was abolished from microtubule plus ends, and dynactin localization was disrupted. Dynactin was found in clumps at the cell cortex along with perturbed astral microtubules. Reducing LIS1 levels with an antisense construct resulted in the accumulation of cells in mitosis and chromosomal abnormalities. NRK cells injected with an antiLIS1 antibody displayed delays in mitotic progression, delayed and unstable chromosome alignment, unattached or mono-oriented chromosomes, and anaphase delay.

These results suggest that LIS1 plays a critical role in cell division and chromosome segregation. They imply that defects seen in human lissencephaly caused by haploinsufficiency of LIS1 may be the result of altered neurogenesis in the proliferative ventricular zone as well as defective migration of neurons into the cortical plate. In support of this, LIS1 dosage reduction in mice is associated with progressive thinning of the cortex, implying that LIS1 may be important for neuronal proliferation (Hirotsune et al. 1998). In addition, proliferation of cells in the ventricular zone of murine Lis1 mutants is disrupted, and embryonic fibroblasts from Lis1 mutants have slow doubling times (M.J. Gambello and A. Wynshaw-Boris, unpubl.).

\section{Cloning of mammalian NudE homologs}

The experiments discussed above provide strong evidence for a functional relationship between LIS1, cytoplasmic dynein, and dynactin in mammalian cells as well as fungi and flies. However, the nature of the physical interactions was unknown until recently. Several groups reported the cloning of mammalian NudE homologs from yeast two-hybrid screens using LIS1 as bait, and some of these studies provided a direct link between LIS1 and dynein motors (Feng et al. 2000; Kitagawa et al. 2000; Niethammer et al. 2000; Sasaki et al. 2000). There are at least two mammalian NudE homologs: mNudE and NUDEL. Both are coiled-coil proteins of 344-345 amino acids and contain 10\% serines. Many of these serines are consensus phosphorylation sites for serinethreonine protein kinases, including Cdk5 (see below), ATM, casein kinase II, and PKCs. As noted above, mNudE and NUDEL proteins display a high degree of homology to each other $(55 \%$ overall identity and $85 \%$ identity in the coiled-coil domain), to nudE from $A$. nidulans (Efimov and Morris 2000), to the nuclear distribution protein RO11 in Neurospora crassa (Minke et al. 1999), and to the mitotic protein MP43 in Xenopus laevis (Stukenberg et al. 1997). The NUDEL protein is heterogeneous, with multiple bands between $42-45 \mathrm{kD}$ detected in the brain (Niethammer et al. 2000; Sasaki et al. 2000), suggesting that it can be phosphorylated (see below), but mNudE was detected as only a single $40-\mathrm{kD}$ band in brain (Feng et al. 2000). LIS1 was immunoprecipitated from brain extracts with either anti-NUDEL or anti-mNudE antibodies, demonstrating a strong in vivo interaction between LIS1 and the two NudE homologs.

mNudE and NUDEL each bind to themselves and to LIS1 via the coiled-coil region (Feng et al. 2000; Niethammer et al. 2000; Sasaki et al. 2000), suggesting that hetero- and homodimerization can occur. The binding sites for LIS1 and mNudE are distinct in the coiled-coil region (Y. Feng and C. Walsh, pers. comm.). LIS1 binding sites for other proteins could not be precisely determined, as only proteins with small truncations from the $\mathrm{N}$ terminus displayed any binding activity. Nearly the entire region of LIS1 consists of the WD repeat domain except for the $\mathrm{N}$-terminal region, so the integrity of this domain may be crucial for normal LIS1 function or protein stability. Point mutations in LIS1 corresponding to those detected in human patients with lissencephaly (LoNigro et al. 1997) severely affected the interaction of LIS1 with itself, NUDEL, and mNudE (Feng et al. 2000; Sasaki et al. 2000), consistent with the functional importance of these interactions. 
There is some overlap in the embryonic patterns of expression of NUDEL and mNudE, but these two genes and proteins also have distinct spatial and temporal patterns of expression, suggesting that they may have distinct functions. NUDEL is expressed in increasing levels from the early embryo to birth and in the adult, especially in the brain (Niethammer et al. 2000; Sasaki et al. 2000). However, mNudE expression is high in the embryo but low after birth in most tissues including the brain (Feng et al. 2000). NUDEL and mNudE proteins colocalize with LIS1 at the centrosome or MTOC in mitotically dividing embryonic neuroblasts or fibroblasts (Feng et al. 2000; Niethammer et al. 2000; Sasaki et al. 2000). Thus, in mitotically dividing cells, LIS1 colocalizes with mNudE and NUDEL.

Once neurons mature, the MTOC undergoes morphological changes and participates in axonal projections and dendrite formation (Bass 1999). As neurons develop in vivo or in vitro, the distribution pattern of NUDEL and LIS1 together began to shift from the MTOC to the axon, and in the adult cortex, it was specifically localized to the axon (Sasaki et al. 2000). In primary neuronal cultures, the initial centrosomal distribution of LIS1 and NUDEL was lost, and these proteins localized to the axon, growth plate, and soma (Sasaki et al. 2000). Elegant studies using deconvolution microscopy using primary neuronal cultures demonstrated that NUDEL colocalized with the dynein IC near the cell body, while NUDEL colocalized with $\mathrm{p} 150^{\text {glued }}$ in the growth cones (Niethammer et al. 2000). Similar experiments have not been done with mNudE.

In the adult, NUDEL and LIS1 are associated with dynein motors during retrograde transport (Sasaki et al. 2000). After ligation of the sciatic nerve, axonal proteins associating with a motor protein that moves anterogradely or retrogradely will accumulate at the proximal or proximal and distal region to the ligation, respectively (Okada et al. 1995; Hanlon et al. 1997). Six hours after ligation, LIS1, NUDEL, and the dynein IC (a retrograde motor protein) accumulated at both sides of the ligation. KIF5C (an anterograde motor protein) accumulated only at the proximal side of the ligation, as expected. These data support the interpretation that NUDEL and LIS1 are associated with the minus-end directed dynein motor during retrograde transport. It is unknown if $\mathrm{mNudE}$ is associated with motor proteins involved in axonal transport.

\section{NUDEL and LIS1 regulate the function of cytoplasmic dynein motors}

The cell biological experiments described above demonstrated that LIS1 and NUDEL were associated with dynein motor proteins in cells but did not prove a physical interaction. Biochemical studies were performed to confirm these studies in neurons. A taxol-treated microtubule fraction that contained the dynein IC from brain contained a significant fraction of tightly associated LIS1 and NUDEL (Sasaki et al. 2000). All three proteins were released by $\mathrm{Mg}$-ATP treatment, which releases dynein motor proteins, suggesting that LIS1 and NUDEL are tightly associated with dyneins. Similarly, LIS1 and NUDEL were contained in insoluble fractions enriched for membrane and cytoskeletal proteins (Niethammer et al. 2000), where Cdk5 and its activator p35 were also found (see below).

A direct interaction between both NUDEL and LIS1 with the CDHC was demonstrated by directed yeast two-hybrid assays and coimmunoprecipitation of relevant proteins expressed by transfection (Sasaki et al. 2000). NUDEL and LIS1 interact with P1-loop domain of CDHC (a region containing the ATP binding site), while only NUDEL interacted with the C-terminal domain of CDHC. Curiously, a distinct region outside of the coiled-coil domain of NUDEL (the region known to be important for self-association and interaction with LIS1) was found to be critical for binding the P1-loop domain and C-terminal domain of CDHC. LIS1-binding sites for NUDEL or the CDHC proteins have not been determined precisely. Thus, LIS1 and NUDEL bind directly and specifically with CDHC and provide a direct biochemical link between LIS1 and cytoplasmic dynein motors.

Niethammer et al. (2000) demonstrated that NUDEL distribution was affected by LIS1 dosage in fibroblasts. Overexpressing LIS1 resulted in tighter perinuclear distribution of NUDEL, while Lis1 +/KO fibroblasts displayed a more diffuse localization of NUDEL. Similarly, Sasaki et al. (2000) showed that NUDEL distribution was affected in embryonic fibroblasts from mice with progressively reduced levels of LIS1 from $50 \%$ to $35 \%$ of wild-type levels. However, the opposite effect on NUDEL distribution was observed: As LIS1 dosage decreased, NUDEL became more tightly associated around the nucleus, as did several proteins associated with CDHC function such as LIS1 itself and $\beta$-tubulin. Similar to previous studies (Smith et al. 2000), reduction of LIS1 resulted in a more diffuse pattern of $\beta$-COP localization, a Golgi complex-associated protein. The reasons for these discrepancies are unclear. It is possible that cells were at different passages or grown under different conditions. In addition, two different antibodies to NUDEL were used. Although these details remain to be resolved, overall, these results suggest that LIS1 functions to localize NUDEL and CDHC /with associated proteins) to regulate dynein motor function and organelle transport in the cell.

If NUDEL also is important for localizing CDHC, then interference with NUDEL function by transfecting cells with constructs expressing full-length or truncated NUDEL proteins should result in a similar mislocalization pattern of dynein motors in the cell. COS cell expression of a C-terminal NUDEL fragment (257-345 amino acids [aa]) that binds to the P1-loop and $\mathrm{C}$ terminus of the CDHC resulted in a similar perinuclear redis tribution of CDHC as observed in cells with reduced levels of LIS1 (Sasaki et al. 2000). Full-length NUDEL or an N-terminal NUDEL (1-256 aa) fragment that binds to NUDEL and LIS1 did not affect CDHC distribution. Although far from conclusive, these results suggest that 
the binding of NUDEL to CDHC regulates its localization and its motor function. Of interest, overexpressed NUDEL colocalized with dynein IC at the cell periphery, while overexpressed LIS1 did not (Niethammer et al. 2000), suggesting that LIS1 and NUDEL may have distinct functions in the regulation of dynein motors. Overall, these results are consistent with the hypothesis that LIS1 and NUDEL are responsible for the localization of $\mathrm{CDHC}$ (with associated proteins) and regulate dynein motor function and organelle transport in the cell. Further expression studies, mouse knock-outs, and perhaps even in vitro dynein motor assays will be required to definitively understand the role of LIS1 and NUDEL in regulating dynein motor function.

\section{mNudE interacts with dynein LC and functions at the centrosome}

Similar to NUDEL, mNudE is localized to the centrosome. LIS1 and NUDEL together bind to CDHC, and this interaction presumably places these proteins with the $\mathrm{CDHC}$ at the centrosome. In contrast, it appears that mNudE alone binds to $\gamma$-tubulin and several other centrosomal proteins (Feng et al. 2000), as mNudE, but not LIS1, can be immunoprecipitated with antibodies to $\gamma$-tubulin. mNudE also interacts in yeast two-hybrid assays with the centrosomal proteins pericentrin, mitosin/ CENP-F, TCP-1, CEP110 and SLAP and the dynein LC, suggesting that mNudE is a central component of the centrosome.

To determine an in vivo function for mNudE, the LIS1-binding domain (aa 88-156) of mNudE was injected into the right blastomere of Xenopus embryos at the two- to four-cell stage (Feng et al. 2000). Embryos injected with mRNA for this truncated mNudE displayed smaller anterior structures and smaller eyes on the injected side of the embryo. The retinas were smaller, and lamination was disrupted. The forebrain and midbrain on the injected side were thickened and disorganized, and abnormal optic tract projections into the tectum were observed. These results suggest that mNudE, through interactions with LIS1, plays an important role in migration or lamination of the central nervous system. As noted above, the binding sites for LIS1 and mNudE are distinct in the coiled-coil region (Y. Feng and C. Walsh, pers. comm.). It should be noted, however, that the region of mNudE used in these experiments is similar to the coiled-coil region of NUDEL, so this fragment of mNudE has the potential to disrupt interactions of mNudE, NUDEL, and any other mammalian NudE homolog with LIS1. Nonetheless, this experiment provides the best evidence that mammalian NudE homologs are involved in the development of the brain.

Overexpression of GFP-mNudE in COS cells resulted in disruption of the centrosomal-based microtubule network (Feng et al. 2000). $\gamma$-tubulin dissociated from the centrosome and displayed a disorganized cytosolic pattern. Disordered microtubules appeared to radiate from aggregates of ectopic GFP-mNudE protein and seemed to recruit $\gamma$-tubulin to these aggregates. This cellular phe- notype is clearly distinct from the effect of overexpression of NUDEL in COS cells, which resulted in a redistribution of dynein motor components. These differences may be the result of distinct functions of NUDEL and $\mathrm{mNudE}$ or of differences in levels of overexpression. These issues can be directly addressed when similar NUDEL and mNudE expression constructs are introduced in the same cells and by mouse knock-outs.

\section{NUDEL is a substrate of Cdk5}

The mitotic phosphoprotein 43 (mp43), an ortholog of NUDE in Xenopus, was originally identified as a protein recognized by the phospho-epitope antibody MPM-2 (Stukenberg et al. 1997). mp43 is phosphorylated in a cell cycle-dependent fashion and is a potential substrate of Cdk serine/threonine kinases. In addition, the NUDEL protein extracted from the brain consists of multiple species that could be reduced to a single one by phosphatase treatment, consistent with posttranslational modification by phosphorylation (Niethammer et al. 2000; Sasaki et al. 2000). NUDEL and Cdk5 were enriched in similar brain fractions and colocalized in the cell bodies and growth cones of embryonic cortical neurons (Niethammer et al. 2000). These findings suggested the intriguing possibility that NUDEL may be a substrate of the Cdk5/ p35 and that NUDEL could provide a link between two neuronal migration pathways not previously known to intersect.

NUDEL is a bona fide substrate for Cdk5/p35 in vitro (Niethammer et al. 2000; Sasaki et al. 2000) and in vivo (Niethammer et al. 2000). The latter studies were particularly convincing. First, the slower-migrating NUDEL species detected in brain extract were reduced but not eliminated in Cdk5 single- or p35/p39 double-mutant mice. Cdk5 activity is not detectable in either of these mutants, suggesting that NUDEL is phosphorylated by this kinase complex as well as others. Second, cotransfection of each of five possible consensus serine/threonine sites' mutants (S198A, T219A, S231A, S242A, and T245A) with Cdk5/p35 into COS cells identified the first three sites (123-NUDEL) as substrates for the enzyme complex, and a triple mutant of these sites was not detectably phosphorylated. Third, embryonic cortical neurons transfected with the 123-NUDEL mutant or treated with roscovitine, a Cdk5 inhibitor, induced neuritic swellings or varicosities that contained dynein IC. As noted above, such defects have also been noted in DLis1 deficient neurons in Drosophila (Liu et al. 2000). Fourth, roscovitine decreased endogenous NUDEL staining at the centrosome/MTOC. Finally, NUDEL staining was more punctate in neurons expressing a dominant negative Cdk5 mutant. These data suggest that Cdk5 phosphorylation of NUDEL controls its cellular localization and probably influences dynein motor function.

These same serine residues are conserved in $\mathrm{mNudE}$, suggesting that mNudE may be a target of Cdk5 as well. Unlike NUDEL, there is no evidence that mNudE is present as multiple bands in the brain. However, direct 
in vitro and in vivo experiments can address whether or not mNudE is a substrate for the Cdk5 complex.

\section{LIS1 regulation of dynein motor function and localization}

The recent studies outlined in this review clearly demonstrate that LIS1 regulates dynein motor function and localization of dynein-associated proteins. Some of the proteins that participate with LIS1 have been identified, and others can be inferred based on the known proteins in complex with cytoplasmic dynein or from genetic studies in a number of eukaryotic organisms. Most notably, LIS1 binds two different NudE homologs to regulate dynein motor function and centrosomal localization. At least one of these homologs, NUDEL, is directly phosphorylated by Cdk5 and its coactivators, p35 and p39. It appears that each of these proteins regulates dynein motor function in a positive fashion, as decreasing the activity, decreasing the amount, or eliminating the protein completely results in decreased dynein motor function and mislocalization of dynein components and centrosomal proteins. Thus, the apparent function of the LIS1 pathway is to activate dynein motors and place them in the proper cellular location for function.

\section{A unified nuclear migration pathway?}

On the basis of the cloning of genes that are mutated in human and murine neuronal migration disorders and the understanding of the function of these gene products, there appear to be at least four genetic and biochemical pathways that regulate neuronal migration: first, the RELN pathway; second, the Cdk5 pathway; third, the LIS1 pathway; and fourth, the DCX pathway (Fig. 2). As noted throughout this review, several recent studies tie together what appeared to be distinct pathways to ultimately regulate dynein motor function via LIS1. Cdk5 phosphorylation of NUDEL (and perhaps mNudE) regulates its localization and may regulate the activity of these NudE homologs. These findings intimately link the Cdk5 and LIS1 pathways of neuronal migration. In fact, it is possible that NUDEL (and mNudE) are the major substrates for Cdk5 with respect to neuronal migration. Cables activates Cdk5 through interaction with c-abl and mDab1, which could link the RELN and Cdk5 pathways. Like LIS1, DCX is a microtubule binding protein, and one report describes an interaction between LIS1 and DCX (Caspi et al. 2000).

A plausible model based on these studies can be proposed to unify all of these pathways into a single pathway that regulates nucleokinesis during neuronal migra-

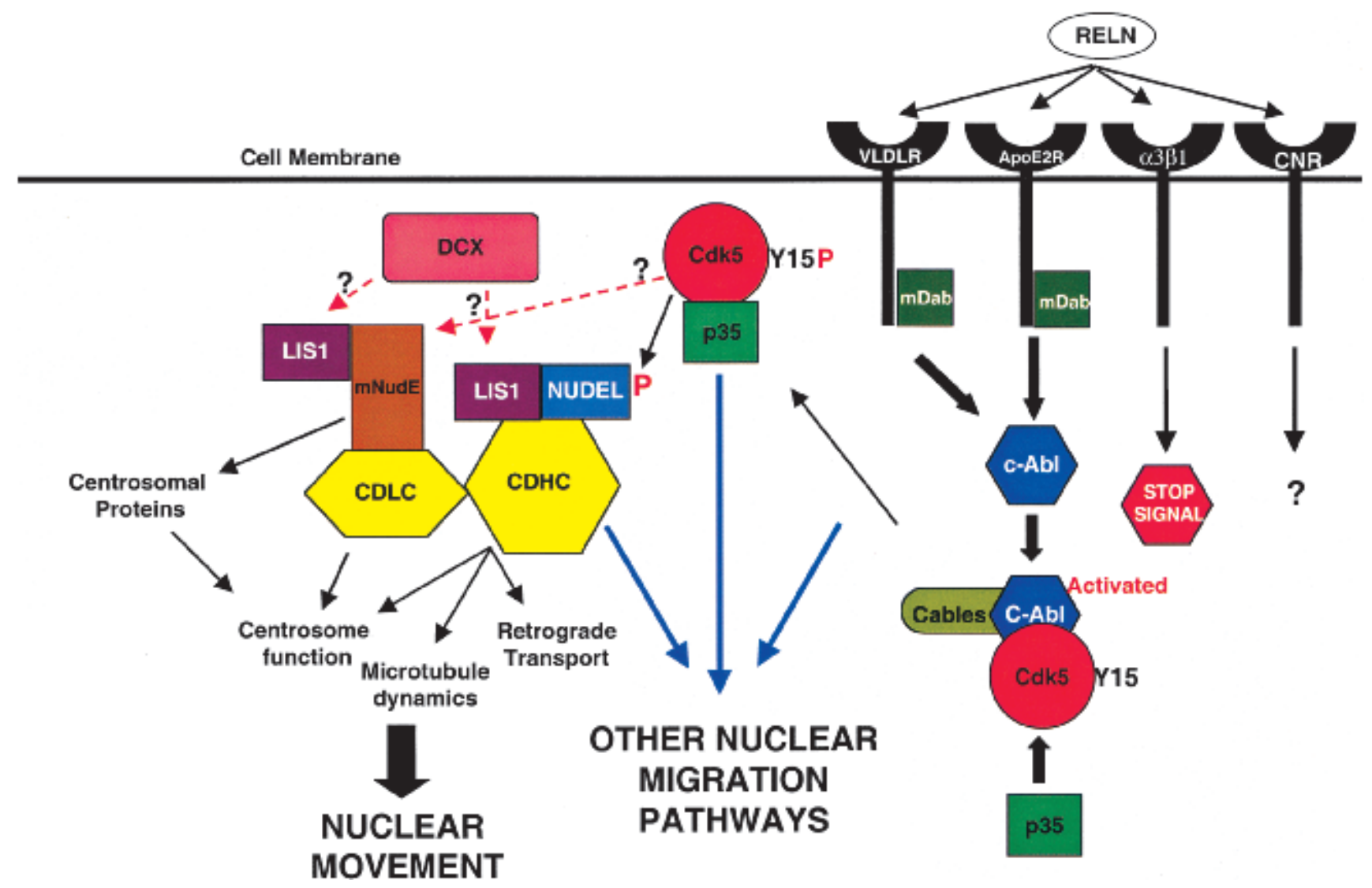

Figure 2. Integration of pathways that regulate nucleokinesis during neuronal migration. The RELN pathway is to the right, Cdk5/ p35 in the middle, and the LIS1 pathway is on the left. Doublecortin (DCX) is placed close to the LIS1 pathway based on its interaction with LIS1 and its role in microtubule dynamics. See text for details. 
tion through the regulation of dynein motor function (Fig. 2). RELN binds to the VLDLR/ApoER2 complex to active mDab1. Activated $\mathrm{mDab} 1$ associates with $\mathrm{c}-\mathrm{abl}$ and activates Cables, which in turn activates the phosphorylation of Cdk5 by c-abl. Activated Cdk5 then phosphorylates NUDEL (and perhaps mNudE). A LIS1-NudE homolog complex then positively regulates dynein motor function through direct association with CDHC and dynein LC. DCX may modulate this process via its association with microtubules and LIS1. On the basis of the conserved pathway in A. nidulans, it is likely that dynein activation will lead at least to the migration of the nucleus into the leading edge during neuronal migration. However, the localization of many of the components of these pathways in the axons and growth cones of neurons also suggests a role for this pathway in leading edge extension.

This model is undoubtedly an oversimplification of what is likely to be a process that is regulated in a highly ordered and complicated fashion. There may be many branch points to and from this basic backbone. For example, it is known that RELN binding to $\alpha 3 \beta 1$-integrin apparently regulates adhesiveness of neurons and provides a stop signal for migration. It is also clear that regulation of the actin cytoskeleton is important for all forms of cell migration, including neuronal migration. However, the relationship among these diverse proteins into a single pathway regulating nucleokinesis during mammalian neuronal migration provides a simple framework for future studies. With the ability to make mice that are mutated in genes corresponding to critical proteins, as well as the current availability of mutants for a number of the proteins in this pathway, the questions can be most directly addressed in the mouse. The use of the mouse will allow examination of genetic interactions in vivo and provide critical cells for in vitro studies. A number of mouse mutants still need to be made or are in the process of being made, including knock-outs and conditional knock-outs for Dcx, Nudel, mNudE, Cables, the Pafah1b catalytic subunits, and conditional mutants for at least some of the dynein subunits. It will also be important to continue to search for proteins that interact with any component of these pathways.

With these tools and information in hand, the outstanding questions that remain can be addressed to understand the mechanisms and processes used to move the nucleus and to extend the leading edge of a migrating neuron. These questions can be addressed by the genetic, neurobiological, and cell biological approaches that have provided us with our current understanding of neuronal migration. What is the precise molecular mechanism of action of LIS1, NUDEL, and mNudE in the regulation and localization of dynein motor function? What is the precise effect of phosphorylation of NUDEL /and perhaps mNudE) on dynein motor function? Does the Cdk5/ NUDEL/LIS1 pathway also regulate proliferation, survival, and axonal transport? Are there adult functions of mNudE? Is there a role for PAFAH1B in regulating LIS1 function in neuronal migration and dynein motor func- tion? How does DCX influence neuronal migration, and does it act via LIS1? Do other kinases than c-abl activate Cdk5? Do other kinases phosphorylate and regulate NUDEL and mNudE function? Does Cdk5 phosphorylate other proteins involved in neuronal migration? Is Cables the only link between Cdk5 and the RELN pathway? Does the RELN pathway activate migration pathways distinct from Cdk5? The answers to these questions will expand our current understanding of the pathways that regulate neuronal migration in mammalian development. This understanding is still in its rudimentary stage, but the convergence of these recent studies provide important connections that can now be experimentally exploited.

\section{Acknowledgments}

We thank Shinji Hirotsune, Joe Gleeson, Li-Huei Tsai, Chris Walsh and Jessica Yingling for insightful discussions and comments on the manuscript.

\section{References}

Barkovich, A.J., Koch, T.K., and Carrol, C.L. 1991. The spectrum of lissencephaly: Report of ten patients analyzed by magnetic resonance imaging. Ann. Neurol. 30: 139-146.

Bass, P.W. 1999. Microtubules and neuronal polarity: Lessons from mitosis. Neuron 22: 23-31.

Beckwith, S.M., Roghi, C.H., Liu, B., and Morris, N.R. 1998. The "8-kD" cytoplasmic dynein light chain is required for nuclear migration and for dynein heavy chain localization in Aspergillus nidulans. J. Cell Biol. 143: 1239-1247.

Caspi, M., Atlas, R., Kantor, A., Sapir, T., and Reiner, O. 2000. Interaction between LIS1 and doublecortin, two lissencephaly gene products. Hum. Mol. Genet. 9: 2205-2213.

Caviness, Jr., V.S. and Rakic, P. 1978. Mechanism of cortical development: A review from mutations in mice. Ann. Rev. Neurosci. 1: 297-326.

Chae, T., Kwon, Y.T., Bronson, R., Dikkes, P., Li, E., and Tsai, L.H. 1997. Mice lacking p35, a neuronal specific activator of Cdk5, display cortical lamination defects, seizures, and adult lethality. Neuron 18: 29-42.

Chiu, Y.-H., Xiang, X., Dawe, A.L., and Morris, R.N. 1997. Deletion of nudC, a nuclear migration gene of Aspergillus nidulans, causes morphological and cell wall abnormalities and is lethal. Mol. Biol. Cell. 8: 1735-1749.

Chong, S.S., Pack, S.D., Roschke, A.V., Tanigami, A., Carrozzo, R., Smith, A.C., Dobyns, W.B., and Ledbetter, D.H. 1997. A revision of the lissencephaly and Miller-Dieker syndrome critical regions in chromosome 17p13.3. Hum. Mol. Genet. 6: $147-155$.

Clark, S.W. and Meyer, D.I. 1992. Centractin is an actin homologue associated with the centrosome. Nature 359: 246-250.

D'Arcangelo, G. and Curran, T. 1998. Reeler: New tales on an old mutant mouse. Bioessays 20: 235-244.

D’Arcangelo, G., Miao, G.G., Chen, S.C., Soares, H.D., Morgan, J.I., and Curran, T. 1995. A protein related to extracellular matrix proteins deleted in the mouse mutant reeler. Nature 374: 719-723.

D'Arcangelo, G., Nakajima, K., Miyata, T., Ogawa, M., Mikoshiba, K., and Curran, T. 1997. Reelin is a secreted glycoprotein recognized by the CR-50 monoclonal antibody. J. Neurosci. 17: 23-31. 
D'Arcangelo, G., Homayouni, R., Keshvara, L., Rice, D., Sheldon, M., and Curran, T. 1999. Reelin is a ligand for lipoprotein receptors. Neuron 24: 471-479.

de Cuevas, M., Lee, J.K. and Spradling, A.C. 1996. $\alpha$-spectrin is required for germline cell division and differentiation in the Drosophila ovary. Development 122: 3959-3968.

des Portes, V., Pinard, J.M., Billuart, P., Vinet, M.C., Koulakoff, A., Carrie, A., Gelot, A., Dupuis, E., Motte, J., Berwald-Netter, Y., et al. 1998. A novel CNS gene required for neuronal migration and involved in X-linked subcortical laminar heterotopia and lissencephaly syndrome. Cell 92: 51-61.

Dobyns, W.B. and Truwit, C.L. 1995. Lissencephaly and other malformations of cortical development: 1995 update. Neuropediatrics 26: 132-147.

Dobyns, W.B., Stratton, R.F., and Greenberg, F. 1984. Syndromes with lissencephaly. I. Miller-Dieker and NormanRoberts syndromes and isolated lissencephaly. Am. J. Med. Genet. 18: 509-526.

Dobyns, W.B., Elias, E.R., Newlin, A.C., Pagon, R.A., and Ledbetter, D.H. 1992. Causal heterogeneity in isolated lissencephaly. Neurology 42: 1375-1388.

Dobyns, W.B., Carrozzo, R., and Ledbetter, D.H. 1994. Frequent deletions of the LIS1 gene in classic lissencephaly. Ann. Neurol. 36: 489-490.

Dobyns, W.B., Andermann, E., Andermann, F., Czapansky-Beilman, D., Dubeau, F., Dulac, O., Guerrini, R., Hirsch, B., Ledbetter, D.H., Lee, N.S., et al. 1996. X-linked malformations of neuronal migration. Neurology 47: 331-339.

Dulabon, L., Olson, E.C., Taglienti, M.G., Eisenbluth, S., McGrath, B., Walsh, C.A., Kriedberg, J.A., and Anton, E.S. 2000. Reelin binds $\alpha 3 \beta 1$-integrin and inhibits neuronal migration. Neuron 27: 33-44.

Efimov, V.P. and Morris, N.R. 2000. The LIS1-related NUDF protein of Aspergillus nidulans interacts with the coiled-coil domain of the NUDE/RO11 protein. J. Cell. Biol. 150: 681688.

Fan, S.S. and Ready, D.F. 1997. Glued participates in distinct microtubule-based activities in Drosophila eye development. Development 124: 1497-1507.

Faulkner, N.E., Dujardin, D.L., Tai, C., Vaughan, K.T., O'Connell, C.B., Wang, Y., and Vallee, R.B. 2000. A role for the lissencephaly gene LIS1 in mitosis and cytoplasmic dynein function. Nat. Cell Biol. 2: 784-791.

Feng, Y., Olson, E.C., Stukenberg, P.T., Flanagan, L.A., Kirschner, M.W., and Walsh, C.A. 2000. LIS1 regulates CNS lamination by interacting with $\mathrm{mNudE}$, a central component of the centrosome. Neuron 28: 665-679.

Fleck, M.W., Hirotsune, S., Gambello, M.J., Phillips-Tansy, E., Suarez, G., Mervis, R.F., Gleeson, J.G., Wynshaw-Boris, A., and McBain, C.J. 2000. Hippocampal abnormalities in Lis1 mutant mice provide a neuronal basis for epileptogenesis in neuronal migration defects. J. Neurosci. 20: 2439-2450

Francis, F., Koulakoff, A., Boucher, D., Chafey, P., Schaar, B., Vinet, M.C., Friocourt, G., McDonnell, N., Reiner, O., Kahn, A., et al. 1999. Doublecortin is a developmentally regulated, microtubule-associated protein expressed in migrating and differentiating neurons. Neuron 23: 247-256.

Garcia-Higuera, I., Fenoglio, J., Li, Y., Lewis, C., Panchenko, M.P., Reiner, O., Smith, T.F., and Neer, E.J. 1996. Folding of proteins with WD-repeats: Comparison of six members of the WD-repeat superfamily to the G protein $\beta$ subunit. Biochemistry 35: 13985-13994.

Gilmore, E.C., Oshima, T., Goffinet, A.M., Kulkarni, A.B., and Herrup, K. 1998. Cyclin-dependent kinase 5-deficient mice demonstrate novel developmental arrest in cerebral cortex. J. Neurosci. 18: 6370-6377.
Giniger, E. 1998. A role for Abl in Notch signaling. Neuron 20: $667-681$

Gleeson, J.G. and Walsh, C.A. 2000. Neuronal migration disorders: From genetic diseases to developmental mechanisms. Trends Neurosci. 23: 352-359.

Gleeson, J.G., Allen, K.M., Fox, J.W., Lamperti, E.D., Berkovic, S., Scheffer, I., Cooper, E.C., Dobyns, W.B., Minnerath, S.R., Ross, M.E., et al. 1998. Doublecortin, a brain-specific gene mutated in human X-linked lissencephaly and double cortex syndrome, encodes a putative signaling protein. Cell 92: 6372 .

Gleeson, J.G. Lin, P.T., Flanagan, L.A., and Walsh, C.A. 1999. Doublecortin is a microtubule-associated protein and is expressed widely by migrating neurons. Neuron 23: 257-271.

Goffinet, A. 1984. Events governing organization of postmigratory neurons: Studies on brain development in normal and reeler mice. Brain Res. Rev. 7: 261-296.

Hanlon, D.W., Yang, Z., and Goldstein, L.S. 1997. Characterization of KIFC2, a neuronal kinesin superfamily member in mouse. Neuron 18: 439-451.

Hattori, M., Adachi, H., Tsujimoto, M., Arai, H., and Inoue, K. 1994. Miller-Dieker lissencephaly gene encodes a subunit of brain platelet-activating factor acetylhydrolase. Nature 370: 216-218.

Hiesberger, T., Trommsdorff, M., Howell, B., Goffinet, A., Mumby, M., Cooper, J., and Herz, J. 1999. Direct binding of Reelin to VLDL receptor and ApoE receptor 2 induces tyrosine phosphorylationof the adapror protein Disabled-1 and modulates $\tau$ phosphorylation. Neuron 24: 481-489.

Hirotsune, S., Takahara, T., Sasaki, N., Hirose, K., Yoshiki, A., Ohashi, T., Kusakabe, M., Murakami, Y., Muramatsu, M., Watanabe, S., et al. 1995. The reeler gene encodes a protein with an EGF-like motif expressed by pioneer neurons. Nat. Genet. 10: 77-83.

Hirotsune, S., Fleck, M.W., Gambello, M.J., Bix, G.J., Chen, A., Clark, G.D., Ledbetter, D.H., McBain, C.J., and WynshawBoris, A. 1998. Graded reduction of Pafah1b1 (Lis1) activity results in neuronal migration defects and early embryonic lethality. Nat. Genet. 19: 333-339.

Holleran, E.A., Karki, S., and Holzbaur, E.L. 1998. The role of the dynactin complex in intracellular motility. Int. Rev. Cytol. 182: 69-109.

Horesh, D., Sapir, T., Francis, F., Wolf, S.G., Caspi, M., Elbaum, M., Chelly, J., and Reiner, O. 1999. Doublecortin, a stabilizer of microtubules. Hum. Mol. Genet. 8: 1599-1610.

Howell, B.W., Hawkes, R., Soriano, P., and Cooper, J.A. 1997a. Neuronal position in the developing brain is regulated by mouse disabled-1. Nature 389: 733-737.

Howell, B.W., Gertler, F.B., and Cooper, J.A. 1997b. Mouse disabled (mDab1): A Src binding protein implicated in neuronal development. ЕMBO J. 16: 1165-1175.

Kitagawa, M., Umezu, M., Aoki, J., Koizumi, H., Arai, H., and Inoue, K. 2000. Direct association of LIS1, the lissencephaly gene product, with a mammalian homologue of a fungal nuclear distribution protein, rNUDE. FEBS Lett. 479: 57-62.

Lee, T. and Luo, L. 1999. Mosaic analysis with a repressible neurotechnique cell marker for studies of gene function in neuronal morphogenesis. Neuron 22: 451-461.

Lees-Miller, J.P., Helfman, D.M., and Schroer, T.A. 1992. A vertebrate actin-related protein is a component of a multisubunit complex involved in microtubule-based vesicle motility. Nature 359: 244-246.

Lin, H., Yue, L., and Spradling, A.C. 1994. The Drosophila fusome, a germline-specific organelle, contains membrane skeletal proteins and functions in cyst formation. Development 120: 947-956. 
Liu, Z., Xie, T., and Steward, R. 1999. Lis1, the Drosophila homologue of a human lissencephaly disease gene, is required for germline cell division and oocyte differentiation. Development 126: 4477-4488.

Liu, Z., Steward, R., and Luo, L. 2000. Drosophila Lis1 is required for neuroblast proliferation, dendritic elaboration and axonal transport. Nat. Cell Biol. 2: 767-775.

Lo Nigro, C., Chong, C.S., Smith, A.C., Dobyns, W.B., Carrozzo, R., and Ledbetter, D.H. 1997. Point mutations and an intragenic deletion in LIS1, the lissencephaly causative gene in isolated lissencephaly sequence and Miller-Dieker syndrome. Hum. Mol. Genet. 6: 157-164.

McGrail, M. and Hays, T.S. 1997. The microtubule motor cytoplasmic dynein is required for spindle orientation during germline cell divisions and oocyte differentiation in Drosophila. Development 124: 2409-2419.

McGrail, M., Gepner, J., Silvanovich, A., Ludmann, S., Serr, M., and Hays, T.S. 1995. Regulation of cytoplasmic dynein function in vivo by the Drosophila Glued complex. J. Cell Biol. 131: 411-425.

Minke, P.F., Lee, I.H., Tinsley, J.H., Bruno, K.S., and Piamann, M. 1999. Neurospora crassa ro-10 and ro-11 genes encode novel proteins required for nuclear distribution. Mol. Microbiol. 32: 1065-1076.

Morris, N.R. 1975. Mitotic mutants of Aspergillus nidulans. Genet. Res. 26: 237-254.

2000. Nuclear migration: From fungi to the mammalian brain. J. Cell Biol. 148: 1097-1101.

Neer, E.J., Schmidt, C.J., Nambudripad, R., and Smith, T. 1994. The ancient regulatory-protein family of WD-repeat proteins. Nature 371: 297-300.

Niethammer, M., Smith, D.S., Ayala, R., Peng, J., Ko, J., Lee, M., Morabito, M., and Tsai, L. 2000. NUDEL is a novel Cdk5 substrate that assocciates with LIS1 and cytoplasmic dynein. Neuron 28: 697-711.

Oakley, B.R. and Morris, N.R. 1980. Nuclear movement is $\beta$-tubulin-dependent in Aspergillus nidulans. Cell 19: 255-262. . 1981. A $\beta$-tubulin mutation in Aspergillus nidulans that blocks microtubule function without blocking assembly. Cell 24: 837-845.

Ogawa, M., Miyata, T., Nakajima, K., Yagyu, K., Seike, M., Ikenaka, K., Yamamoto, H., and Mikoshiba, K. 1995. The reeler gene-associated antigen on Cajal-Retzius neurons is a crucial molecule for laminar organization of cortical neurons. Neuron 14: 899-912.

Okada, Y., Yamazaki, H., Sekine-Aizawa, Y., and Nobutaka, H. 1995. The neuron-specific kinesin superfamily protein KIF1A is a unique monomeric motor for antegrade axonal transport of synaptic vesicle precursor. Cell 81: 769-780.

Oshima, T., Ward, J.M., Huh, C.G., Longnecker, G., Veeranna, A., Pant, H.C., Brady, R.O., Martin, L.J., and Kulkarni, A.B. 1996. Targeted disruption of the cyclin-dependent kinase 5 gene results in abnormal corticogenesis, neuronal pathology and perinatal death. Proc. Natl. Acad. Sci. 93: 11173-11178.

Osmani, A.H., Osmani, S.A., and Morris, N.R. 1990. The molecular cloning and identification of a gene product specifically required for nuclear movement in Aspergillus nidulans. J. Cell. Biol. 111: 543-551.

Paylor, R., Hirotsune, S., Gambello, M.J., Yuva-Paylor, L., Crawley, J., and Wynshaw-Boris, A. 1999. Impaired learning and motor behavior in heterozygous Pafah1b1 (Lis1) mutant mice. Learn. Mem. 6: 521-537.

Pilz, D.T., Matsumoto, N., Minnerath, S., Mills, P., Gleeson, J.G., Allen, K.M., Walsh, C.A., Barkovich, A.J., Dobyns, W.B., Ledbetter, D.H., et al. 1998. LIS1 and XLIS (DCX) mutations cause most classical lissencephaly, but different pa- terns of malformation. Hum. Mol. Genet. 7: 2029-2037.

Reiner, O., Carrozzo, R., Shen, Y., Wehnert, M., Faustinella, F., Dobyns, W.B., Caskey, C.T., and Ledbetter, D.H. 1993. Isolation of a Miller-Dieker lissencephaly gene containing G protein $\beta$-subunit-like repeats. Nature 364: 717-721.

Rice, D.S. and Curran, T. 1999. Mutant mice with scrambled brains: Understanding the signaling pathways that control cell positioning in the CNS. Genes \& Dev. 13: 2758-2773.

Sasaki, S., Shionoya, A., Ishida, M., Gambello, M., Yingling, J., Wynshaw-Boris, A., and Hirotsune, S. 2000. A LIS1/NUDEL/ cytoplasmic dynein heavy chain complex in the developing and adult central nervous system. Neuron 28: 681-696.

Sapir, T., Elbaum, M., and Reiner, O. 1997. Reduction of microtubule catastrophe events by LIS1, platelet-activating factor acetylhydrolase subunit. EMBO J. 16: 6977-6984.

Schafer, D.A., Gill, S.R., Cooper, J.A., Heuser, J.E., and Schroer, T.A. 1994. Ultrastructural analysis of the dynactin complex: An actin-related protein is a component of a filament that resembles F-actin. J. Cell Biol. 126: 403-412.

Schroer, T.A. 1994. New insights into the interaction of cytoplasmic dynein with the actin-related protein, Arp1. J. Cell Biol. 127: 1-4.

Senzaki, K., Ogawa, M., and Yagi, T. 1999. Proteins of the CNR family are multiple receltors for Reelin. Cell 99: 635-647.

Sheffield, P.J., Garrard, S., Caspi, M., Aoki, J., Arai, H., Derewenda, U., Inoue, K., Suter, B., Reiner, O., and Derewenda, Z.S. 2000. Homologs of the $\alpha$ - and $\beta$-subunits of mammalian brain platelet-activating factor acetylhydrolase Ib in the Drosophila melanogaster genome. Proteins 39: 1-8.

Sheldon, M., Rice, D.S., D'Arcangelo, G., Yoneshima, H., Nakajima, K., Mikoshiba, K., Howell, B.W., Copper, J.A., Goldowitz, D., and Curran, T. 1997. Scramber and yotari disrupt the disabled gene and produce a reeler-like phenotype. $\mathrm{Na}$ ture 389: 730-733.

Smith, D.S., Niethammer, M., Zhou, Y., Gambello, M.J., Wynshaw-Boris, A., and Tsai, L.-H. 2000. Regulation of cytoplasmic dynein behavior and mirotubule organization by mammalian Lis1. Nat. Cell Biol. 2: 767-775.

Stukenberg, P.T., Lustig, K.D., McGarry, T.J., King, R.W., Kuang, J., and Kirschner, M.W. 1997. Systematic identification of mitotic phoshoproteins. Curr. Biol. 7: 338-348.

Swan, A., Nguyen, T., and Suter, B. 1999. Drosophila Lissencephaly-1 functions with Bic-D and dynein in oocyte determination and nuclear positioning. Nat. Cell Biol. 1: 444449.

Trommsdorff, M., Gorrhardt, M., Hiesberger, T., Shelton, J., Stockinger, W., Nimpf, J., Hammer, R., Richardson, J., and Herz, J. 1999. Reeler/Disabled-like disruption of neuronal migration in kcockout mice lacking the VLDL receptor and ApoE receptor 2. Cell 97: 689-701.

Walsh, C.A. and Goffinet, A.M. 2000. Potential mechanisms of mutations that affect neuronal migration in man and mouse. Curr. Opin. Genet. Dev. 10: 270-274.

Wang, D.S., Shaw, R., Hattori, M., Arai, H., Inoue, K., and Shaw, G. 1995. Binding of pleckstrin homology domains to WD40/ $\beta$-transducin repeat containing segments of the protein product of the Lis-1 gene. Biochem. Biophys. Res. Commun. 209: 622-629.

Ware, M.L., Fox, J.W., Gonzalez, J.L., Davis, N.M., Lambert de Rouvroit, C., Russo, C.J., Chua, Jr., S.C., Goffinet, A.M., and Walsh, C.A. 1997. Aberrant splicing of a mouse disabled homolog, mdabl, in the scrambler mouse. Neuron 19: 239-249.

Willins, D.A., Liu, B., Xiang, X., and Morris, N.R. 1997. Mutations in the heavy chain of cytoplasmic dynein suppress the nudF nuclear migration mutation of Aspergillus nidulans. Mol. Gen. Genet. 255: 194-200. 
Xiang, X. and Morris, N.R. 1999. Hyphal tip growth and nuclear migration. Curr. Opin. Microbiol. 2: 636-640.

Xiang, X., Beckwith, S.M., and Morris, N.R. 1994. Cytoplasmic dynein is involved in nuclear migration in Aspergillus nidulans. Proc. Natl. Acad. Sci. 91: 2100-2104.

Xiang, X., Osmani, A.H., Osmani, S.A., Xin, M., and Morris, N.R. 1995. NudF, a nuclear migration gene in Aspergillus nidulans, is similar to the human LIS- 1 gene required for neuronal migration. Mol. Biol. Cell 6: 297-310.

Xiang, X., Zuo, W., Efimov, V.P., and Morris, N.R. 1999. Isolation of a new set of Aspergillus nidulans mutants defective in nuclear migration. Curr. Genet. 35: 626-630.

Yue, L. and Spradling, A.C. 1992. hu-li tai shao, a gene required for ring canal formation during Drosophila oogenesis, encodes a homolog of adducin. Genes \& Dev. 6: 2443-2454.

Zukerberg, L.R., Patrick, G.N., Nikolic, M., Humbert, S., Wu, C.L., Lanier, L.M., Gertler, F.B., Vidal, M., Van Etten, R.A., and Tsai, L.H. 2000. Cables links Cdk5 and c-Abl and facilitates Cdk5 tyrosine phosphorylation, kinase upregulation, and neurite outgrowth. Neuron 26: 633-646. 


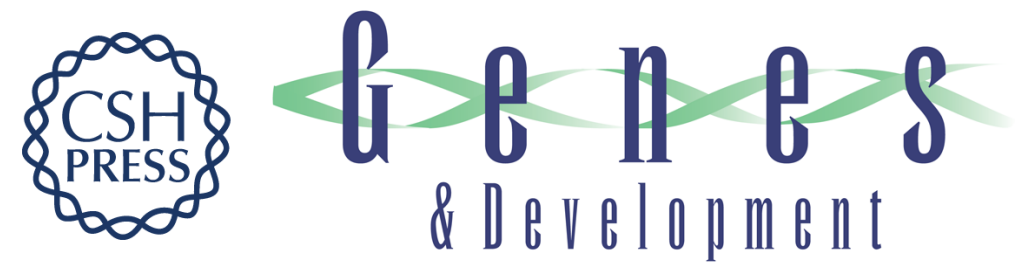

\section{LIS1 and dynein motor function in neuronal migration and development}

Anthony Wynshaw-Boris and Michael J. Gambello

Genes Dev. 2001, 15:

Access the most recent version at doi:10.1101/gad.886801

References This article cites 87 articles, 25 of which can be accessed free at: http://genesdev.cshlp.org/content/15/6/639.full.htmI\#ref-list-1

License

Email Alerting

Receive free email alerts when new articles cite this article - sign up in the box at the top Service right corner of the article or click here.

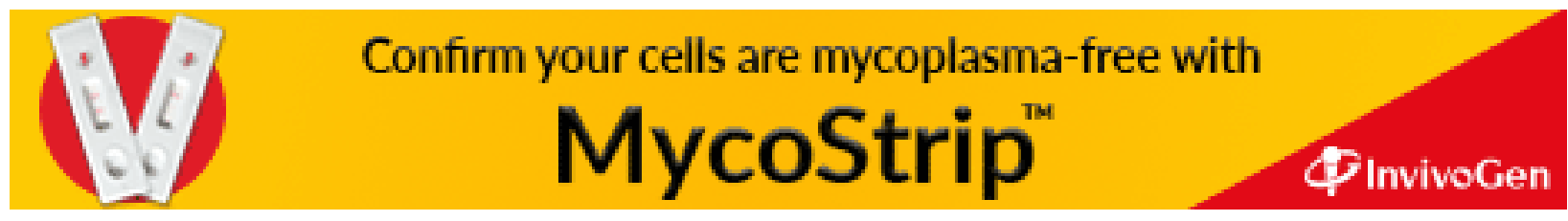

